\title{
Entendendo a Motivação para o Uso de um Ambiente Virtual de Aprendizagem: Um Survey com base no Modelo TAM3 e na Teoria da Autodeterminação
}

\author{
Natália J. S. de Oliveira ${ }^{1,2}$, Sean W. M. Siqueira ${ }^{1}$ \\ ${ }^{1}$ Programa de Pós-Graduação em Informática - Universidade Federal do Estado do Rio \\ de Janeiro (UNIRIO) - Rio de Janeiro - RJ - Brasil \\ ${ }^{2}$ Escola de Ciência e Tecnologia - Universidade do Grande Rio (UNIGRANRIO) - Rio \\ de Janeiro - RJ - Brasil \\ \{natalia.oliveira,sean\}@uniriotec.br, natalia_joana@unigranrio.edu.br
}

\begin{abstract}
Motivation may directly influence users' decisions about whether using or not an information system. There exist many models for evaluating use and acceptance of technology, but motivational aspects are not fully exploited in most of them. The objective of this work was to identify factors and the motivation type that influence the use and acceptance of a Virtual Learning Environment. We employed TAM3 (Technology Acceptance Model 3) and Self-Determination Theory in a survey in a private university. Results evidence that students are more extrinsically than intrinsically motivated and that their motivation decreases as the course progresses.
\end{abstract}

Resumo. A motivação pode influenciar diretamente na decisão do usuário em utilizar ou não um sistema de informação. Existem diversos modelos para avaliar o uso e a aceitação de tecnologias, mas aspectos motivacionais não são totalmente explorados na maioria. O objetivo deste trabalho foi identificar os fatores e o tipo de motivação que influenciam o uso e a aceitação de um ambiente virtual de aprendizagem. Foi utilizado o Modelo TAM3 (Technology Acceptance Model 3) e a Teoria da Autodeterminação em um survey em uma universidade privada. Os resultados confirmam que os alunos se encontram mais motivados extrinsecamente do que intrinsecamente e que, ao longo do curso, vão se sentindo mais desmotivados.

\section{Introdução}

Por ser a "peça-chave" das atividades humanas, a motivação vem sendo objeto de estudo em diversas áreas de conhecimento. No contexto dos Sistemas de Informação, a motivação pode influenciar diretamente a satisfação do usuário e, consequentemente, a sua decisão de utilizar ou não um determinado sistema. Um estudo realizado por Delone e Mclean (2003) mostrou que "Motivação" e "Modelo de Uso e Aceitação de Tecnologias" estão intimamente ligados aos Sistemas de Informação, uma vez que o "uso levará a uma maior satisfação do usuário, da mesma forma que o aumento da satisfação do usuário levará ao aumento da intenção de usar e, assim, usar." 
VII Congresso Brasileiro de Informática na Educação (CBIE 2018)

Anais do XXIX Simpósio Brasileiro de Informática na Educação (SBIE 2018)

Tais modelos relacionados a motivação, bem como ao uso e à aceitação de tecnologias, podem ser empregados em diversas áreas do conhecimento, inclusive na Educação, desde que sejam devidamente adaptados. Assim, o objetivo deste trabalho é identificar os fatores e o tipo de motivação que influenciam o uso e a aceitação de um Ambiente Virtual de Aprendizagem (AVA). Para tanto, o Modelo de Aceitação de Tecnologia 3 (TAM3 - Technology Acceptance Model 3) foi escolhido para ser utilizado nesta pesquisa, por ser o mais completo em relação às questões motivacionais.

A Teoria da Autodeterminação (SDT - Self-Determination Theory) foi escolhida por ser amplamente utilizada e referenciada quando se trata de motivação. Foi elaborada em 1981 e especifica diferentes tipos de motivação, sendo baseada nas diferentes razões que dão origem a uma ação. Como o nome indica, autodeterminação está relacionada com a "necessidade que as pessoas têm de serem autodeterminadas, ou seja, de se sentirem no controle de suas próprias ações". Tal teoria faz uma distinção clássica entre as motivações intrínseca e extrínseca: a intrínseca ocorre quando a pessoa se motiva por ela mesma, ou seja, pelo seu próprio interesse (auto motivada). A extrínseca, por outro lado, ocorre quando a pessoa é motivada por recompensas ou outros estímulos externos (Deci e Ryan, 1985).

A SDT pode ser resumida através de um continuum formado por seis tipos de motivação (Desmotivação, Externa, Introjetada, Identificada, Integrada e Intrínseca) que variam qualitativamente desde a desmotivação, passando por vários níveis de motivação extrínseca, até chegar à intrínseca. Oliveira e Siqueira (2018) apresentam uma discussão sobre como combinar diferentes teorias relacionadas à motivação. A SDT se destaca das outras teorias motivacionais existentes devido ao processo de internalização. De acordo com Ryan e Deci (2000), "quanto mais internalizamos e assimilamos as razões de uma ação, mais nossas ações extrinsecamente motivadas se tornam autodeterminadas".

Apesar do TAM3 contemplar aspectos motivacionais, não é o suficiente para determinar o tipo de motivação que está levando ou não o usuário a aceitar e usar um determinado sistema. Por isso, após estudo aprofundado da literatura realizado com o objetivo de alcançarmos melhor entendimento dos conceitos inerentes aos diversos modelos e teorias existentes, sentimos a necessidade de complementar o questionário do TAM3 com questões relativas à SDT (Ryan e Connell, 1989). Deste modo, um questionário foi elaborado e aplicado a um grupo de alunos que utilizam o Blackboard $^{l}$ como AVA em uma universidade privada. O Blackboard foi implantado recentemente na Instituição, que resolveu investir em cursos de capacitação para que seus professores pudessem explorar da melhor maneira possível os recursos que o ambiente tem a oferecer. Acreditamos que a combinação do TAM3 e SDT facilita a mensuração dos efeitos de determinadas variáveis e o entendimento dos motivos que levam o aluno a aceitar e usar o AVA considerado.

Este artigo é organizado da seguinte forma: a seção 2 descreve sucintamente os principais modelos de uso e aceitação de tecnologia existentes; a seção 3 apresenta trabalhos relacionados ao tema de pesquisa; a seção 4 apresenta a descrição do survey realizado e os resultados obtidos; e a seção 5 apresenta as conclusões.

\footnotetext{
${ }^{1} \mathrm{http}: / /$ www.blackboard.com/index.html
} 
VII Congresso Brasileiro de Informática na Educação (CBIE 2018)

Anais do XXIX Simpósio Brasileiro de Informática na Educação (SBIE 2018)

\section{Teorias e Modelos de Uso e Aceitação de Tecnologias}

Os modelos de uso e aceitação de tecnologias vêm sendo amplamente estudados e aplicados em todo o mundo desde 1989. De acordo com Oliveira e Martins (2011), os modelos e teorias mais utilizados são (siglas em inglês): Modelo de Aceitação de Tecnologia - TAM (Davis, 1989); Teoria do Comportamento Planejado - TPB (Ajzen, 1991); Teoria Unificada de Aceitação e Uso de Tecnologia - UTAUT (Venkatesh et al., 2003); Teoria da Difusão da Inovação - DOI (Rogers, 1995); e Tecnologia, Organização e Ambiente - TOE (Tornatzky et al., 1990). Além de ser o mais amplamente adotado, o TAM considera aspectos relacionados à motivação desde sua primeira versão.

O TAM é baseado na Teoria da Ação Racionalizada (TRA - Theory of Reasoned Action), que é uma das mais importantes e influentes teorias do comportamento humano (Fishbein e Ajzen, 1975). O modelo foi desenvolvido com o intuito de entender o que leva um indivíduo a aceitar ou rejeitar a Tecnologia da Informação. Ele é formado por dois constructos teóricos que são considerados os determinantes fundamentais para o uso de um sistema (Davis, 1989):

- Utilidade Percebida (PU - Perceived Usefulness): Grau em que uma pessoa acredita que utilizar determinado sistema melhora o seu desempenho nas atividades.

- Facilidade de Uso Percebida (PEOU - Perceived Ease of Use): Grau em que uma pessoa acredita que a utilização de determinado sistema será fácil.

Venkatesh e Davis (2000) estenderam o TAM, originando o TAM2, que inclui novos constructos que detalham melhor a PU em termos de processo de influência social (norma subjetiva, voluntariedade e imagem) e processos instrumentais cognitivos (relevância no trabalho, qualidade de resultados, demonstrabilidade do resultado e facilidade de uso percebida) (Venkatesh e Davis, 2000):

- Norma Subjetiva (SN - Subjective Norm): Grau em que um indivíduo percebe que a maioria das pessoas consideradas importantes para ele pensa se ele deveria ou não usar o sistema.

- Imagem (IMG - Image): Grau em que um indivíduo percebe que o uso de uma inovação aumentará seu status em seu sistema social.

- Relevância do Trabalho (REL - Job Relevance): Grau em que um indivíduo acredita que o sistema é aplicável ao seu trabalho.

- Qualidade de Saída (OUT - Output Quality): Grau em que um indivíduo acredita que o sistema executa bem suas tarefas.

- Demonstrabilidade dos Resultados (RES - Result Demonstrability): Grau em que um indivíduo acredita que os resultados do uso do sistema são tangíveis, observáveis e comunicáveis.

Da combinação entre o TAM2 e o modelo dos determinantes da facilidade de uso percebida (Venkatesh, 2000), surgiu a mais nova versão do TAM: o TAM3, proposto por Venkatesh e Bala (2008). Esta versão inclui novos constructos que detalham melhor a PEOU. Os constructos são: controle (interno e externo - definidos como "auto eficácia 
VII Congresso Brasileiro de Informática na Educação (CBIE 2018)

Anais do XXIX Simpósio Brasileiro de Informática na Educação (SBIE 2018)

do computador" e "percepção de controle externo", respectivamente), motivação intrínseca (definida como "ludicidade") e emoção (definida como "ansiedade em utilizar o computador"). Além disso, Venkatesh (2000) também considera importante para este novo modelo a inclusão de constructos de ajuste: a "usabilidade objetiva" e o "prazer percebido". Ainda nesta versão, os autores testaram a relação entre a variável "experiência" e os constructos "ansiedade em utilizar o computador", "facilidade de uso percebida" e "intenção comportamental", o que não havia ocorrido nos modelos anteriores. Os determinantes do Modelo TAM3 são (Venkatesh e Bala, 2008):

- Auto Eficácia do Computador (CSE - Computer Self-Efficacy): Grau em que um indivíduo acredita ter a capacidade de realizar uma tarefa/trabalho específico usando o computador.

- Percepção de Controle Externo (PEC - Perception of External Control): Grau em que um indivíduo acredita existirem recursos organizacionais e técnicos para apoiar o uso do sistema.

- Ansiedade em utilizar o Computador (ANX - Computer Anxiety): Grau de apreensão de um indivíduo, ou mesmo medo, quando ele se depara com a possibilidade de usar computadores.

- Ludicidade (CPLAY - Computer Playfulness): Grau de espontaneidade cognitiva nas interações com microcomputadores.

- Prazer Percebido (ENJ - Perceived Enjoyment): Até que ponto "a atividade de usar um sistema específico é percebida como agradável por si só, além de quaisquer consequências de desempenho resultantes do uso do sistema".

- Usabilidade Objetiva (OU - Objective Usability): Comparação de sistemas com base no nível real (em vez de percepções) do esforço necessário para completar tarefas específicas.

Em relação aos demais modelos citados, o TAM3 é o único que trata mais explicitamente as questões motivacionais e, por isso, fundamenta este trabalho. Davis (1989) relata que a motivação intrínseca estava começando a ser reconhecida já na primeira versão do TAM como um mecanismo de grande potencial por trás da aceitação pelo usuário. No TAM3, os autores consideram a "utilidade percebida" como uma crença instrumental que é "conceitualmente semelhante à motivação extrínseca" (Venkatesh e Bala, 2008) e, consequentemente, pode-se concluir que a "facilidade de uso percebida" está associada à motivação intrínseca.

\section{Trabalhos Relacionados}

O TAM vem sendo amplamente estudado na área de e-learning. Abdullah e Ward (2016) realizaram uma revisão sistemática a fim de identificar os fatores externos mais comuns nos trabalhos que ampliaram o modelo TAM para sustentar a aceitação ou o uso do e-learning num período de dez anos. Foram identificados 152 fatores externos diferentes em 107 trabalhos. Dentre os fatores, apenas cinco foram encontrados em dez ou mais estudos e por isso foram considerados os mais comuns. São eles: auto eficácia, norma subjetiva, prazer, ansiedade por usar o computador e experiência. 
VII Congresso Brasileiro de Informática na Educação (CBIE 2018)

Anais do XXIX Simpósio Brasileiro de Informática na Educação (SBIE 2018)

Lin et al. (2014) e Ramirez-Anormaliza et al. (2015) utilizaram o TAM para investigar a aceitação de AVAs. Mbarek e Zaddem (2013) e Zanini (2016) utilizaram como base o modelo TAM para identificar a importância da interação no ambiente de $e$ learning. Al-sayyed (2015) e Balog (2015) utilizaram o TAM3 para investigar a aceitação do e-learning em universidades. Nota-se, entretanto, que nenhum destes trabalhos utilizou a SDT.

A combinação entre TAM e SDT vem sendo explorada na literatura em diferentes contextos. Recentemente, Cheng et al. (2016) utilizaram esta combinação para criar um modelo de motivação capaz de identificar as motivações dos usuários no contexto de "economia compartilhada" (e.g., aluguel, empréstimo, permuta) através de uma plataforma digital. Akhlaq e Ahmed (2013) os combinaram para identificar se os fatores motivacionais extrínsecos e/ou intrínsecos aumentam ou diminuem a confiança para a adoção do internet banking.

No contexto educacional, esta combinação também está presente. Roca e Gagné (2008) propuseram uma extensão do TAM no contexto de e-learning a fim de analisar as relações entre as variáveis da SDT e as variáveis do TAM e avaliar a intenção dos usuários em continuar ou não a fazer cursos de e-learning fora do ambiente de trabalho. Para isto, introduziram autonomia, competência e pertencimento (relação) como determinantes da utilidade percebida, da ludicidade percebida e da facilidade de uso percebida. Prieto et al. (2014) também ampliaram o TAM com constructos de outras teorias para avaliar a aceitação de tecnologias móveis, tendo professores como foco.

No entanto, o grande diferencial de nosso estudo é a utilização do modelo TAM3 combinado com a SDT especificamente para avaliar o uso e aceitação de AVA. Além disso, o questionário foi complementado com questões relacionadas a jogos, interação e gamificação. A gamificação consiste na utilização de elementos de jogos (e.g., troféus, medalhas, avatar) em ambientes fora do contexto de jogos com o objetivo de motivar os indivíduos a uma determinada ação, auxiliar na solução de problemas e promover aprendizagem (Deterding e Dixon, 2011; Werbach e Hunter, 2012). Os elementos de jogos também podem ser classificados como intrínsecos e extrínsecos, como pode ser visto no trabalho de Lopes et al. (2015).

\section{Descrição do Survey}

A fim de avaliar o uso e aceitação de AVA por alunos, foi conduzido um survey com um grupo de 160 alunos de uma universidade privada. Os alunos são da modalidade presencial, onde $20 \%$ das disciplinas obrigatórias são oferecidas a distância com apoio de um AVA. O grupo era composto por alunos dos cursos de Tecnologia da Informação TI (Sistemas de Informação, Análise de Sistemas e Redes de Computadores) e Engenharias (Civil e de Produção). A coleta de dados foi realizada na última semana do semestre 2018/1 na presença do professor. A participação foi voluntária e nenhum incentivo foi oferecido. Todas as respostas foram anônimas e confidenciais. Com isso, obtivemos um total de 59 voluntários respondentes (36,8\% do total disponível), sendo 34 alunos de TI e 25 alunos de Engenharia. 
VII Congresso Brasileiro de Informática na Educação (CBIE 2018)

Anais do XXIX Simpósio Brasileiro de Informática na Educação (SBIE 2018)

Os dados foram coletados através de um questionário ${ }^{2}$ onde os alunos informaram o curso e o período, bem como responderam 73 questões, usando uma escala Likert de sete pontos, variando de "discordo totalmente" a "concordo totalmente". Vale ressaltar que, embora o questionário tenha utilizado como base o TAM3, este precisou ser adaptado ao contexto do estudo. Das 73 questões, 50 eram referentes ao modelo TAM3, 7 representavam os tipos de motivação presentes na SDT e 16 procuravam entender o que estava faltando ao AVA para que se tornasse mais atraente. Nesse sentido, foram incluídas então questões relacionadas a jogos, interação e gamificação, como, por exemplo, pontos extras, classificação dos resultados, recompensas, entre outras.

A Tabela 1 apresenta a média geral, a mediana, o desvio padrão e o grau de concordância obtidos para cada um dos constructos que compõem o modelo TAM3. Observa-se que a "intenção comportamental", a "auto eficácia do computador" e a "ansiedade em utilizar o computador" apresentaram números mais elevados dentre todos os constructos analisados. Nota-se ainda que o desvio padrão apresentado para todos os constructos mostra que houve uma dispersão nas respostas dadas pelos participantes.

Tabela 1. Média geral dos constructos que compõem o TAM3

\begin{tabular}{|c|c|c|c|c|c|}
\hline Sigla & Constructo & Média & Mediana & $\begin{array}{c}\text { Desvio } \\
\text { Padrão }\end{array}$ & $\begin{array}{c}\text { Grau de } \\
\text { Concordância }\end{array}$ \\
\hline PU & Utilidade Percebida & 3,758475 & 4 & 1,711419 & Neutro \\
\hline PEOU & Facilidade de Uso Percebida & 3,911017 & 4 & 1,840149 & Neutro \\
\hline SN & Norma Subjetiva & 4,293785 & 4 & 1,792519 & Neutro \\
\hline VOL & Voluntariedade & 3,214689 & 3 & 1,853142 & Discordo Parcialmente \\
\hline IMG & Imagem & 2,847458 & 2 & 1,644854 & Discordo \\
\hline REL & Relevância do Trabalho & 4,231638 & 4 & 1,882874 & Neutro \\
\hline OUT & Qualidade de Saída & 3,734463 & 4 & 1,776367 & Neutro \\
\hline RES & Demonstrabilidade dos Resultados & 4,508475 & 5 & 1,713584 & Discordo Parcialmente \\
\hline BI & Intenção Comportamental & 4,146893 & 4 & 2,017131 & Neutro \\
\hline CSE & Auto Eficácia do Computador & 4,483051 & 5 & 1,966814 & Neutro \\
\hline PEC & Percepção de Controle Externo & 4,427966 & 4 & 1,895328 & Neutro \\
\hline CPLAY & Ludicidade & 3,300847 & 3 & 1,638571 & Discordo Parcialmente \\
\hline CANX & Ansiedade em Utilizar o Computador & 3,838983 & 4 & 2,086967 & Neutro \\
\hline ENJ & Prazer Percebido & 3,022599 & 3 & 1,762621 & Discordo Parcialmente \\
\hline & Média Geral & & & $\mathbf{3 , 8 3 7 1 6 7}$ & \\
\hline
\end{tabular}

No geral, os constructos que representam o modelo TAM3 se mantiveram na neutralidade. A "demonstrabilidade dos resultados" foi o único constructo em que houve concordância por parte dos alunos, o que indica que acreditam que os resultados do uso do AVA são "tangíveis, observáveis e comunicáveis". Na média, o constructo "auto eficácia do computador" se manteve neutro, mas a mediana mostrou que os alunos tendem a concordar que são capazes de utilizar o AVA sem precisar de ajuda. Entretanto, a maioria dos alunos discorda quanto às questões referentes a

\footnotetext{
${ }^{2} \mathrm{O}$ questionário completo encontra-se disponível em:

https://drive.google.com/drive/folders/1SJGCZ4p4_QlYXgMXHcCDFSrvaTIMNdBs?usp=sharing
} 
VII Congresso Brasileiro de Informática na Educação (CBIE 2018)

Anais do XXIX Simpósio Brasileiro de Informática na Educação (SBIE 2018)

"voluntariedade", "imagem", "ludicidade" e "prazer percebido". Dentro do modelo TAM3, a "ludicidade" representa explicitamente a motivação intrínseca e, como pode ser visto na Tabela 1, o resultado foi bem abaixo do esperado, o que nos leva a concluir que os alunos não estão usando AVA porque querem usar, mas sim por serem obrigados a fazê-lo. Tal fato também é observado através da análise das questões relacionadas à SDT que foram incluídas para complementar o TAM3 (Figura 1).

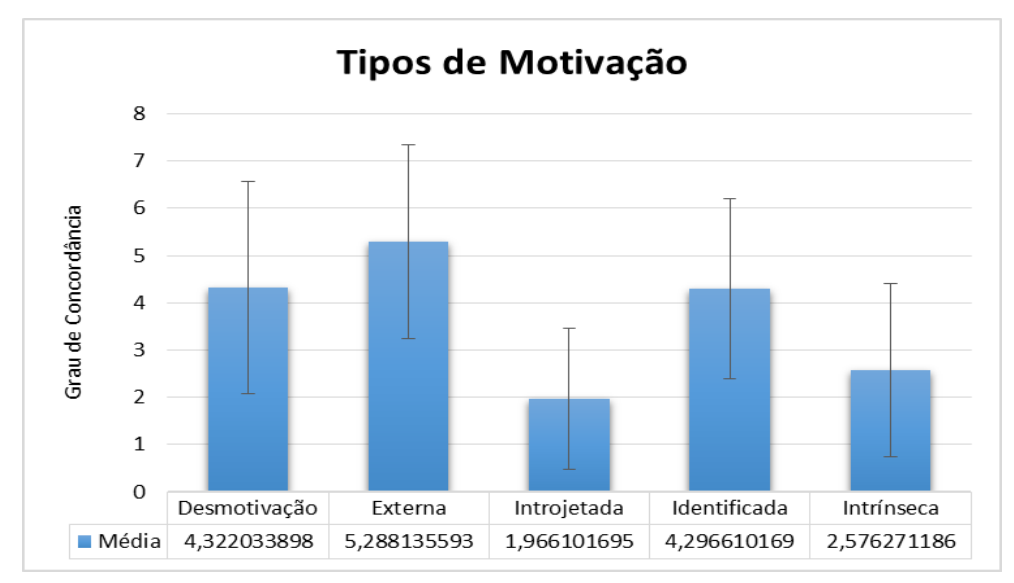

Figura 1. Representação da motivação sob a ótica da Teoria da Autodeterminação

O gráfico mostra que a regulação externa teve o maior nível de concordância $(5,28)$, o que a coloca como o principal tipo de motivação para os alunos. Este é o tipo menos autônomo de motivação, pois se encontra mais próximo da desmotivação do que os demais tipos. Nesse caso, o indivíduo age somente em prol de recompensas ou até mesmo para evitar punições. A desmotivação e a regulação identificada encontram-se praticamente no mesmo nível, se mantendo na neutralidade. A regulação identificada é aquela onde o aluno se identifica com o objetivo, percebe a importância da realização de uma determinada tarefa, ou seja, existe uma certa internalização, mesmo que o motivo para fazê-la ainda seja externo (Ryan e Deci 2000).

Ao analisar os dados considerando o período em que cada aluno se encontra, pode-se perceber uma discrepância significativa em relação às questões da SDT. O survey contou com alunos de diversos períodos $\left(1^{\circ}, 3^{\circ}, 4^{\circ}, 5^{\circ}, 7^{\circ}, 8^{\circ}\right.$ e $\left.10^{\circ}\right)$, que foram divididos da seguinte forma: períodos iniciais $\left(1^{\circ}\right.$ e $\left.3^{\circ}\right)$, períodos intermediários $\left(4^{\circ}\right.$ e $\left.5^{\circ}\right)$ e períodos finais $\left(7^{\circ}, 8^{\circ}\right.$ e $\left.10^{\circ}\right)$. Para os alunos de períodos iniciais, a regulação externa e a regulação identificada se mantiveram com média 5,00; nos demais períodos, a regulação identificada se manteve neutra. Em comparação aos demais períodos, os alunos de períodos iniciais estão sendo menos motivados pela regulação externa e mais motivados pela regulação identificada. Pode-se então concluir que, ao iniciar o curso de graduação, eles utilizam o AVA porque o consideram importante para sua formação e, com o passar do tempo, o processo de internalização é desfeito e a regulação identificada vai perdendo espaço para a regulação externa. Já a análise feita por curso mostrou que os resultados se mantiveram para todas as questões do questionário, não havendo discrepâncias significativas para serem aqui apresentadas.

Em relação ao tempo médio de uso semanal do AVA, 66\% dos alunos informaram que o utilizam apenas para realizar as atividades propostas e nada mais, $17 \%$ utilizam entre 15 minutos e 1 hora e os $17 \%$ restantes utilizam por mais de 2 horas. Para finalizar, os alunos concordam que deveria haver maior interação com professores e 
VII Congresso Brasileiro de Informática na Educação (CBIE 2018)

Anais do XXIX Simpósio Brasileiro de Informática na Educação (SBIE 2018)

tutores e inclusão de elementos de jogos para que o AVA se tornasse mais atraente. Assim como no trabalho de Mbarek e Zaddem (2013), a interação se mostrou um fator crucial para que o AVA se torne mais atrativo para os alunos. Segundo os autores, "o sentimento de interatividade dos aprendizes melhora a presença social e minimiza a sensação de isolamento". Os jogos também podem ajudar a promover a motivação nos estudantes. Silva et al. (2014) afirmam que os jogos "possuem em seu desenvolvimento elementos que garantem a existências da motivação". Neste sentido, algumas questões tiveram um alto índice de concordância:

- $51 \%$ gostariam de desafios valendo pontos extras.

- $46 \%$ gostariam que houvesse feedback de professores e/ou tutores sobre o seu desempenho em cada atividade avaliativa.

- $41 \%$ gostariam que esporadicamente houvesse profissionais atuantes no mercado de trabalho participando das interações.

\section{Conclusão}

O objetivo deste trabalho foi identificar os fatores e os tipos de motivação que influenciam o uso e a aceitação de um AVA em uma universidade privada. Para tanto, foi desenvolvido e aplicado um questionário para os alunos. Trata-se de uma extensão do questionário do TAM3 com conceitos da SDT e de gamificação. A análise dos resultados mostra que tal abordagem foi bastante eficaz, pois foi possível identificar o tipo de motivação que leva o aluno a utilizar o AVA e comprovar o baixo índice da motivação intrínseca descrita pelo TAM3 como "ludicidade". O survey revelou que os constructos do TAM3 "demonstrabilidade dos resultados", "auto eficácia do computador" e "percepção de controle externo" são os que possuem maior influência no uso e aceitação do AVA. Em relação à motivação, os resultados revelam que, com o passar do tempo, os alunos se desmotivam e só utilizam o AVA para realizar tarefas.

Além disso, algumas questões foram elaboradas para auxiliar no entendimento das características que faltam ao AVA para torná-lo mais atrativo. Elas mostraram que faltam incentivo e recompensas por parte da Instituição, ou seja, mais elementos para motivação extrínseca. Este resultado concorda com as crenças dos autores do modelo TAM3. Segundo eles, "o incentivo e uma importante recompensa extrínseca podem reduzir a ansiedade e aumentar o prazer percebido, uma vez que as recompensas são consideradas importantes impulsionadores de motivações intrínsecas" (Venkatesh e Bala 2008).

Este trabalho contribui para o entendimento sobre a motivação e uso de AVA. Como trabalhos futuros, aplicaremos o questionário para alunos de uma diversidade maior de cursos. Investigaremos também a modalidade totalmente a distância e o uso do AVA como ferramenta de apoio para a modalidade presencial. Além disso, seria interessante realizar o mesmo estudo com alunos de diferentes instituições, sejam públicas ou privadas, bem como considerar uma análise da motivação, da aceitação e do uso de diferentes AVA.

\section{Referências}

Abdullah, F., \& Ward, R. (2016). Developing a General Extended Technology 
VII Congresso Brasileiro de Informática na Educação (CBIE 2018)

Anais do XXIX Simpósio Brasileiro de Informática na Educação (SBIE 2018)

Acceptance Model for E-Learning (GETAMEL) by analysing commonly used external factors. Computers in Human Behavior, 56, 238-256.

Ajzen, I. (1991). The Theory of Planned Behavior. Organizational Behavior and Human Decision Processes, 50(2), 179-211.

Akhlaq, A., \& Ahmed, E. (2013). The effect of motivation on trust in the acceptance of internet banking in a low income country. International Journal of Bank Marketing, $31(2), 115-125$.

Al-sayyed, F. S. (2015). A Framework for e-Learning Acceptance: A Case Study of the Palestinian Universities. Faculty of Graduate Studies.

Balog, A. (2015). Acceptance of e-Learning Systems: a Serial Multiple Mediation Analysis. Studies in Informatics and Control, 24(1), 101-110.

Cheng, X., Zhu, R., \& Fu, S. (2016). Modeling the Motivation of Users' Sharing Option: A Case Study Based on A Car-Sharing Digital Platform. Fifteenth Wuhan International Conference on E-Business, 45-52.

Davis, F. D. (1989). Perceived Usefulness, Perceived Ease of Use, and User Acceptance of Information Technology. MIS Quarterly, 13(3), 319.

Deci, E. L., \& Ryan, R. M. (1985). Intrinsic Motivation and Self-Determination in Human Behavior. New York: Plenum Press.

Delone, W., \& Mclean, E. (2003). The DeLone and McLean Model of Information Systems Success: A Ten-Year Update. Journal of Management Information Systems, 19(4), 9-30.

Deterding, S., \& Dixon, D. (2011). From Game Design Elements to Gamefulness: Defining "Gamification." In Proceedings of the 15th international academic MindTrek conference: Envisioning future media environments. ACM (pp. 9-15).

Fishbein, M., \& Ajzen, I. (1975). Belief, attitude, intention, and behavior: An introduction to theory and research. Reading, MA: Addison-Wesley.

Lin, S., Persada, S. F., \& Nadlifatin, R. (2014). A Study of Student Behavior in Accepting the Blackboard Learning System: a Technology Acceptance Model (TAM) Approach. In IEEE 18th International Conference on Computer Supported Cooperative Work in Design (pp. 457-462).

Lopes, R. A., Toda, A. M., \& Brancher, J. D. (2015). Um estudo preliminar sobre elementos extrínsecos e intrínsecos do processo de Gamification. Revista Brasileira de Informática Na Educação (RBIE), 23(3), 164-173.

Mbarek, R., \& Zaddem, F. (2013). The examination of factors affecting e-learning effectiveness. International Journal of Innovation and Applied Studies, 2(4), 423435 .

Oliveira, N. J. S. de, \& Siqueira, S. W. M. (2018). OntoMotivation: Combinando Teorias de Motivação. In XLIV Latin American Computing Conference (CLEI) Latin American Symposium on Computing and Society (SLIS), 2018, São Paulo.

Oliveira, T., \& Martins, M. F. (2011). Literature Review of Information Technology Adoption Models at Firm Level. The Electronic Journal Information Systems 
VII Congresso Brasileiro de Informática na Educação (CBIE 2018)

Anais do XXIX Simpósio Brasileiro de Informática na Educação (SBIE 2018)

Evaluation, 14(1), 110-121.

Prieto, J. C. S., Migueláñez, S. O., \& García-Peñalvo, F. J. (2014). Mobile learning adoption from informal into formal. In Proceedings of the Second International Conference on Technological Ecosystems for Enhancing Multiculturality. ACM (pp. 595-602).

Ramirez-Anormaliza, R., Sabaté, F., \& Guevara-Viejo, F. (2015). Evaluating Student Acceptance Level of E-learning Systems. In International Conference of Education, Research and Innovation. "ICERI2015: Proceedings 8th International Conference of Education, Research and Innovation November 16th-18th (pp. 2393-2399).

Roca, J. C., \& Gagné, M. (2008). Understanding e-learning continuance intention in the workplace: A self-determination theory perspective. Computers in Human Behavior, 24(4), 1585-1604.

Rogers, E. M. (1995). Diffusion of Innovations (4th Editio). New York: The Free Press.

Ryan, R. M., Connell, J. P. (1989). Perceived Locus of Causality and Internalization: Examining Reasons for Acting in Two Domains. Journal of Personality and Social Psychology, 57(5), 749-761.

Ryan, R. M., \& Deci, E. L. (2000). Intrinsic and Extrinsic Motivations: Classic Definitions and New Directions. Contemporary Educational Psychology, 25(1), 5467.

Silva, T. S. C., Tedesco, P. C. D. A. R., \& Melo, J. C. B. De. (2014). A importância da motivação dos estudantes e o uso de técnicas de engajamento para apoiar a escolha de jogos no ensino de programação. In XXV Simpósio Brasileiro de Informática na Educação (pp. 11-15).

Tornatzky, L. G., Fleischer, M., \& Chakrabarti, A. K. (1990). The process of Tecnology Innovation. Lexington Books.

Venkatesh, V. (2000). Determinants of Perceived Ease of Use: Integrating Control, Intrinsic Motivation, and Emotion into the Technology Acceptance Model. Information Systems Research, 11(4), 342-365.

Venkatesh, V., \& Bala, H. (2008). Technology Acceptance Model 3 and a Research Agenda on Interventions. Decision Sciences, 39(2), 273-315.

Venkatesh, V., \& Davis, F. D. (2000). A Theoretical Extension of the Technology Acceptance Model: Four Longitudinal Field Studies. Management Science, 46(2), 186-204.

Venkatesh, V., Morris, M. G., Davis, G. B., \& Davis, F. D. (2003). User Acceptance of Information Technology: Toward a Unified View. MIS Quarterly, 27(3), 425.

Werbach, K., \& Hunter, D. (2012). For the win: How game thinking can revolutionize your business. Wharton Digital Press.

Zanini, A. da S. (2016). Práticas Escolares Mediadas pela Wiki do Moodle: Análise de Aceitação e Percepções dos Estudantes. Dissertação (Mestrado em Educação) Universidade Federal de Santa Maria (UFSM). 